\title{
An image-based gangrene disease classification
}

\author{
Pramod Sekharan Nair'1, Tsrity Asefa Berihu², Varun Kumar ${ }^{3}$ \\ ${ }^{1,2}$ Department of Computing Technology, Aksum University, Ethiopia \\ ${ }^{3}$ Department of Mathematics, Aksum University, Ethiopia
}

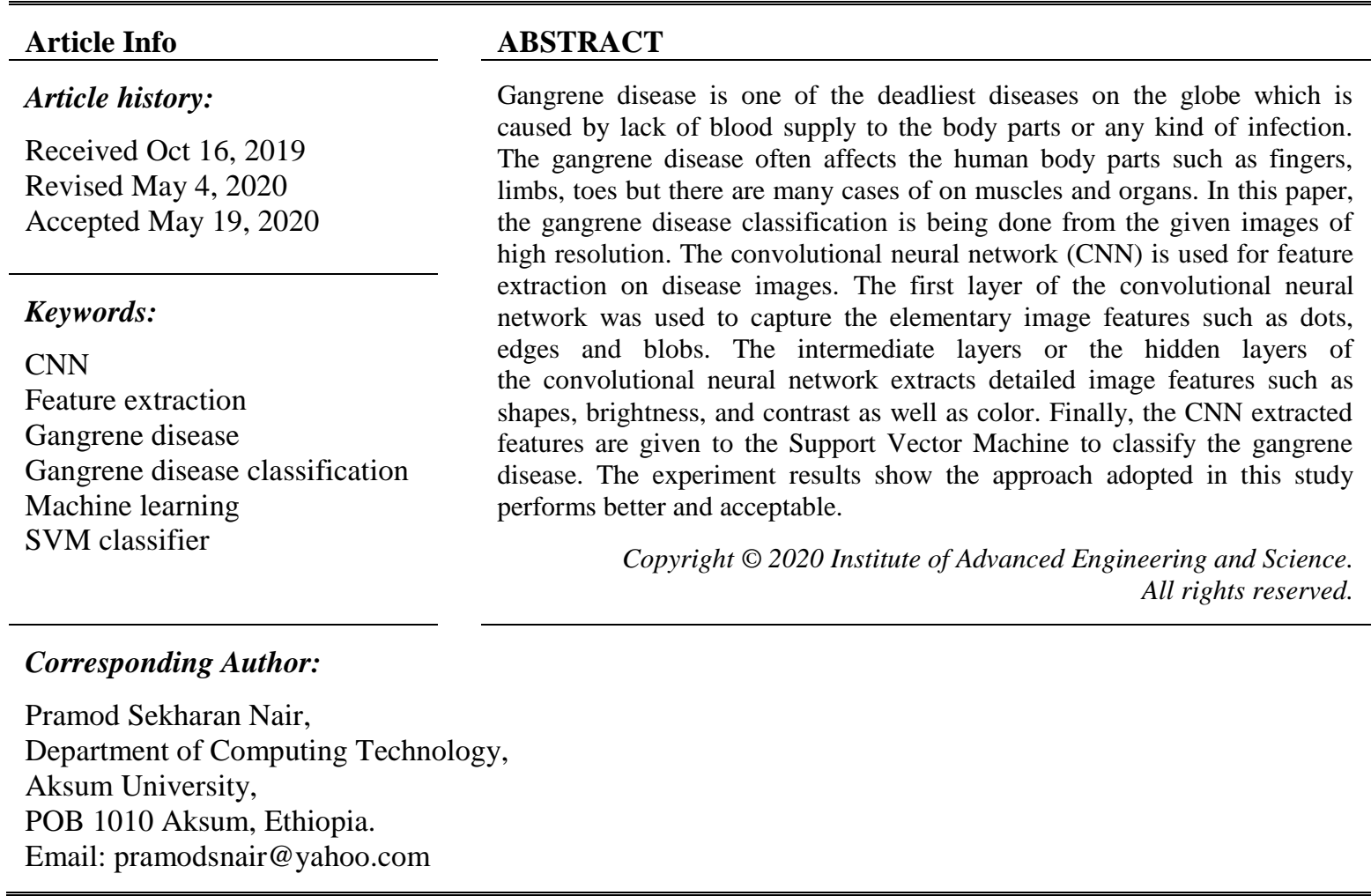

\section{INTRODUCTION}

Gangrene is a disease that results in the deterioration or loss of an organ or tissue usually caused by a deficiency of blood supply in the body. This may be caused by infectious or inflammatory processes because of injury or wounds on body parts, or progressive changes arising from chronic diseases, like diabetes mellitus. Gangrene disease could also result in damage of the tissue caused deficiency of blood supply. The blood supply may cut off to the affected part of the body as a result of it may leads to various problems, including infection, vascular disease or trauma. If this disease is widespread over the body parts, then shock may occur, can even cause death.

Currently, Orthopedists base the symptoms for the diagnosis and classification of Gangrene disease on the visual assessment of the affected part. The expert inspire to detect the disease features or symptoms as the early signs are a numb sensation and coldness in the affected parts, starts to change the color of the body part, usually changing from reddish to brown and then finally changes into black color, the affected part shrivels and becomes dry, swollen and then decays, the wound is infected and contains discharge, resulting in black skin color, kills blood vessels. This fact shows that right diagnosis to gangrene disease highly depends on the experts' experience and on his or her visual insight. In addition to this, the human vision system lacks precision, and quantification in the way gathers information from an image. Recently, most clinical centers in both the developing and developed countries have at least magnetic resonance imaging (MRI), computed tomography (CT) scan and some other expensive clinical laboratory equipments, so it is difficult to serve the community especially in developing countries with the help of MRI and CT scan machines. These machines are not smart enough in identification and classification of Gangrene disease types, this research motivated to automate the human vision system for classification of the disease. To minimize 
the errors made by the physicians during manual diagnosis and classification, there is a need of developing such a prototype for diagnosis and classification of gangrene disease to reduce man made errors during diagnosis of the disease. This research simplifies the classification of the disease and provides consistent and satisfactory way to correctly classify different types of Gangrene diseases. Therefore, our effort is expected to fill existing gaps by developing an image-based hybrid approach for classification of the disease using convolutional neural network (CNN) and support vector machine (SVM). Based on the literature survey, we have identified the following problems in gangrene disease diagnosis: Man made errors are common in diagnosis and classification of gangrene disease when carried out manually. The unavailability of faster diagnosis and classification approach to help the doctor.

\section{LITREATURE REVIEW}

In this section, the works related to gangrene disease diagnosis and classification will be reviewed. There are three types of gangrene disease $[1,2]$ these are:

a. Dry Gangrene disease: This type of gangrene disease is common in people suffering from blood vessel disease or diabetes and autoimmune disease. Usually affects hands, feet and tissue. Infection is typically not present in dry Gangrene. Some of the symptoms are:

- $\quad$ dry and shriveled skin that changes color from blue to black and eventually sloughs off

- clear line of demarcation is seen

- limited to the demarcation

- $\quad$ slow gradual loss of blood supply

- $\quad$ cold and numb skin and

- $\quad$ pain may or may not be present

b. Wet Gangrene [1, 2]: Common in people with wounds or having body part wrinkled or squeezed. In this type of gangrene disease, the affected tissue swells and is called "wet" because of presence of discharge of fluid as a result of the disease. By infection the wet Gangrene spreads rapidly through the body, making wet Gangrene a very serious and potentially life intimidating condition. So, immediate treatment advised. Some of the symptoms are:

- $\quad$ swelling and pain at the site of infection

- change in skin color from red to brown to black

- blisters or sore that produce a bad smelling discharge or pus

- $\quad$ line of demarcation is vague and

- $\quad$ sudden loss of blood supply

c. Gas Gangrene [2]: This type of gangrene disease is rare but more dangerous. The disease occurs when infection progresses deep inside the body parts, like muscles or organs usually as a result of trauma. The bacterium that causes gas Gangrene disease is called clostridia, which releases toxins or poisons that cause damage to all parts of the body along with gas is trapped within the body tissue. As the condition progresses the skin becomes pale and gray and this results in cracking sound when pressed due to the gas accumulation within the tissue. Gas Gangrene disease needs immediate medical treatment because without treatment death may occur within 48 hours.

The Support vector machine [3] is used in this work to do the classification of digital images into either of the categories of gangrene diseases. Support vector machines represent an extension to nonlinear models of the generalized model for classification. The SVM algorithm is based on the statistical machine learning theory [4]. And furthermore, the SVM is based on the concept of decision planes used to define decision boundaries. A decision plane separates between a set of objects having different class memberships. SVM is a set of related supervised learning methods used in classification problems [5] and regression [6]. Classification task is based on drawing separating lines to distinguish between objects of different class memberships known as hyperplane classifiers. Support vector machines are particularly suited to handle such tasks.

SVM is a binary classifier that assumes only two values as labels which are negative and positive. Multiclass SVM is used to classify objects with more than two classes [7]. In our work the multiclass SVM is used for classify different types of gangrene diseases. In one of the works [8], for classifying both melanocytic skin lesions and non-melanocytic skin lesions a new computer-aided method was implemented. As by the author, the method used as four texture features for extraction: Contrast, Correlation, Homogeneity and Energy. The SVM and K-nearest neighbor (K-NN) classification techniques are selected to compare the performance and analyze the accuracy of both classifiers.

Automated classification of liver disorders using ultrasound images [9] was proposed, which shows an innovative method used in recognition of fatty liver disease and heterogeneous liver using textural analysis of liver ultrasound images. The wavelet packet transform was used in the study to extract 
the features such as mean, median and standard deviation, the classification technique used is the multiclass SVM, and an accuracy of $95 \%$ was achieved using the multiclass SVM for classification.

Another study showcased that image processing techniques can be applied to Lung cancer detection [10]. This study was based on the image processing techniques. Furthermore, study shows that, image preprocessing in the early stages of disease diagnosis process, improved the accuracy and the result. In the study, three stage image processing were used: enhancement, segmentation using marker-controlled watershed, feature extraction Gabor filter stage. The watershed segmentation technique had better accuracy $(84.55 \%)$ compared to other approaches $[11,12]$.

The accuracy of computer vision applications is improved [13] with the surge in the usage of convolutional networks and its techniques. A technique based on machine learning and computer vision [14] was used in plant leaf disease classification using CNN [15], which has been utilized for the detection and classification of plant leaf disease. The paper was focused on the early classification and detection of the plant leaf diseases. The authors used a convolutional neural network for feature extraction and classification. The authors did not train the entire deep convolutional neural network (DCNN) from the scratch with a random initialization, instead, pre-train a DCNN on a very large dataset was used and they trained DCNN weights to classify the plant leaf disease for four types of leaves such us Apple, Grape, Corn, and Strawberry. One another work on plant disease classification was proposed [16] by using CNN. This study has used the soybean leaf images to identify three different diseases on soybean. The authors have used the pre trained $\mathrm{CNN}$ for feature extraction and classification of the diseases.

There is another work [17] uses the Siamese CNN, multiple instances of the same model, for classify the x-ray image of the chest to classify the pneumonia disease. This paper was focused on the classification of the x-ray image in to any of the three classes. Two classes were the two different causes of pneumonia and the other one is noninfected $\mathrm{x}$-ray image. The $\mathrm{CNN}$ is widely used in feature extraction from the images. One of the recent works [18] proposes the feature extraction of face images to do the age-invariant face recognition.

In most of the research works [19, 20] related to disease classification, image processing was in the crucial part of disease diagnosis process using machine learning algorithms. Image pre-processing is the first step in image processing and pre-processing helps to improve the quality of the image [21] so that the image is suitable to extract the features. Due to different factors, the image captured by the digital camera may not be clear and in acceptable resolution. Therefore, different pre-processing techniques are applied to images to improve the image quality. This includes removal of noises, adjustment of intensity or brightness and addition of edge detectors based on the preferred features for the classification.

The edge-based segmentation [21] method changes rapidly based on a change in image intensity value and the change in a single intensity value does not deliver better result of edges. Edge detection method is used to locating the edges where either the first derivative of intensity is greater than a particular threshold or the second derivative has zero crossings. The first step in edge-based segmentation is detecting the edges and then connecting them together to make the object boundaries and segment the necessary areas.

In conclusion segmentation is the common image processing method, where an image is divided or partitioned into different constituent parts with similar features like, pixel intensity value, color and texture [22]. The simplest method divides the image pixels with respect to their intensity level. These methods are used on images with brighter objects than their background. The selection of the methods is either manual or automatic based on prior knowledge or information of image features [23, 24].

\section{RESEARCH METHOD}

Interviews were used for data gathering and data collection. Physicians and nurses are interviewed on gangrene disease. The medical images collected for Gangrene disease were classified into three categories of gangrene disease, namely wet Gangrene, dry Gangrene, gas Gangrene. The different types of gangrene disease images are shown in Figure 1. The use of machine learning algorithms to classify medical images into separate categories [25] is nowadays a common computing and data science task. Machine learning algorithms need a special feature extraction tool and a properly prepared dataset. The medical images used in this work which are collected from different image sources were cropped out in uniform resolution of 224-by-224-by-3 to make the dataset to be similar in size and resolution. Preparing a properly labeled dataset with medical images for different types of Gangrene disease is an important step in the classification process. We have conducted interviews with medical experts such as, orthopedists (physicians) to correctly select the medical images for Gangrene disease from specialized hospitals.

The Gangrene classification problem can be solved through a supervised machine learning method. In this work the CNN was used to extract the features from the image dataset. SVM was used to classify the extracted image features into different gangrene disease categories. The architecture of the image-based 
hybrid approach for gangrene disease classification system is shown in Figure 2. MATLAB is used to implement the proposed system.

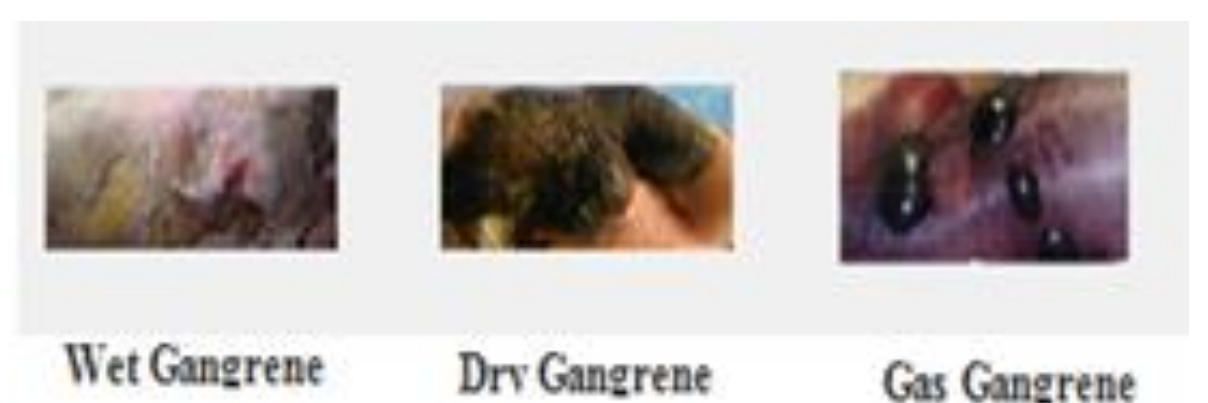

Figure 1. Three types of gangrene disease and their labels

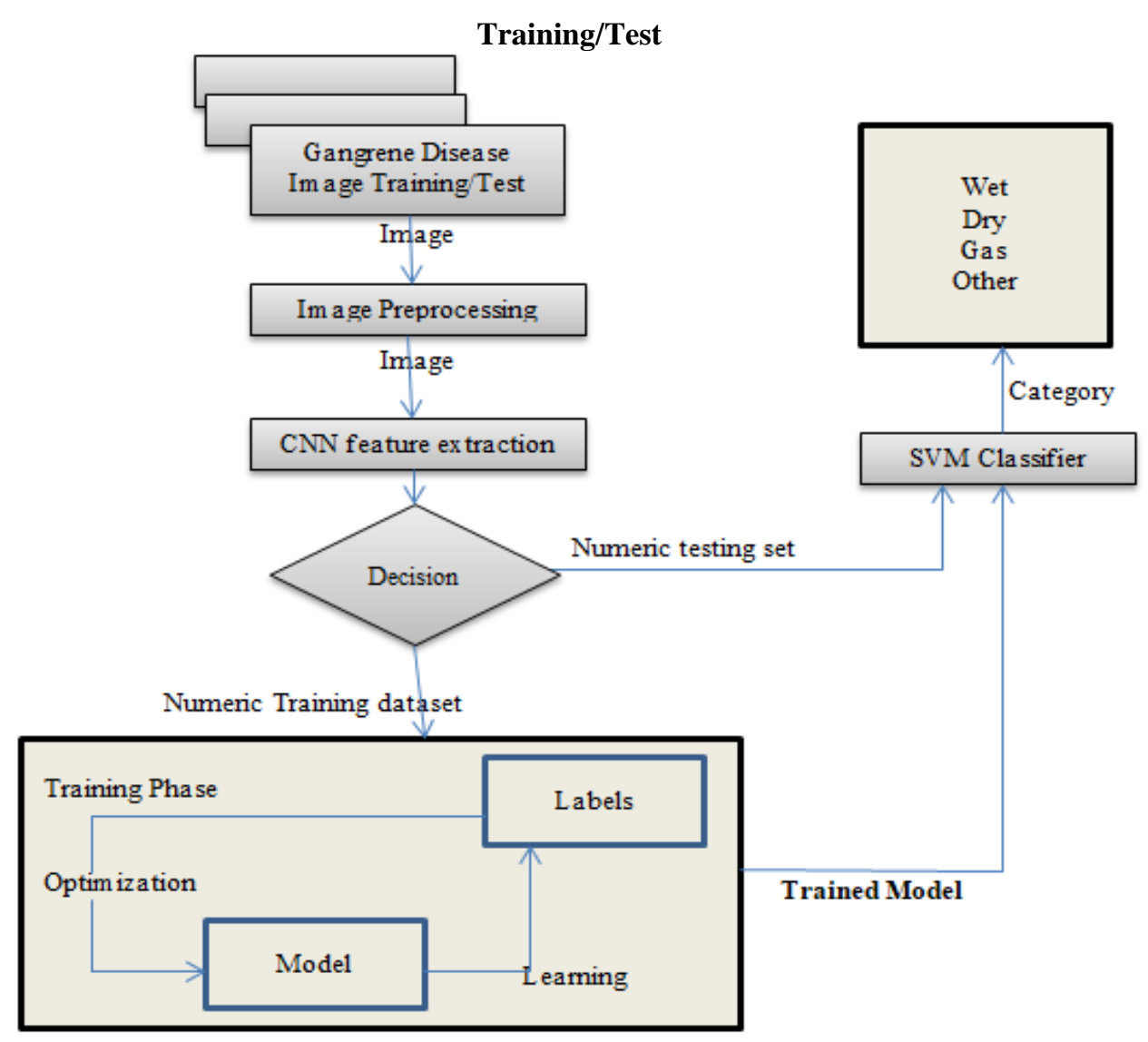

Figure 2. Architecture for gangrene disease classification

\section{RESULTS AND DISCUSSIONS} is discussed.

In this section, the experimental results on performance of SVM classifier with the confusion matrix

\subsection{Confusion matrix of SVM classifier}

The confusion matrix shows in Table 1 is used to showcase the accuracy of SVM classifier on a set of test data set for which the observed values of gangrene disease images are known. The outputs from the confusion matrixs are shown in Tables 1(a) and 1(b). The Figure 2 shows the graphical explanation of the tables. 
Since there are three classes of Gangrene diseases and the fourth one that for not a gangrene disease, confusion matrix for the classes were generated. But, to measure the overall performance and accuracy of the SVM classifier the aggregate of the precision and recall from the SVM were considered. The implemented classifier only generates the final confusion matrix and the corresponding average accuracy as shown in Tables 1(a) and 1(b).

Table 1. (a) Confusion matrix tabulated according to

\begin{tabular}{ccccc}
\multicolumn{5}{c}{ the true positive } \\
\hline Number & Wet & Dry & Gas & Other \\
\hline Wet & 8 & 0 & 0 & 2 \\
Dry & 2 & 7.3 & 0 & 0.6 \\
Gas & 0 & 0 & 10 & 0 \\
Other & 1.3 & 0 & 0 & 8.6 \\
\hline
\end{tabular}

Table 1. (b) Confusion matrix tabulated according to

\begin{tabular}{ccccc}
\multicolumn{5}{c}{ the true negative } \\
\hline Number & Wet & Dry & Gas & Other \\
\hline Wet & 0.8000 & 0 & 0 & 0.2000 \\
Dry & 0.2000 & 0.7333 & 0 & 0.0667 \\
Gas & 0 & 0 & 1.000 & 0 \\
Other & 0.1333 & 0 & 0 & 0.8667 \\
\hline
\end{tabular}

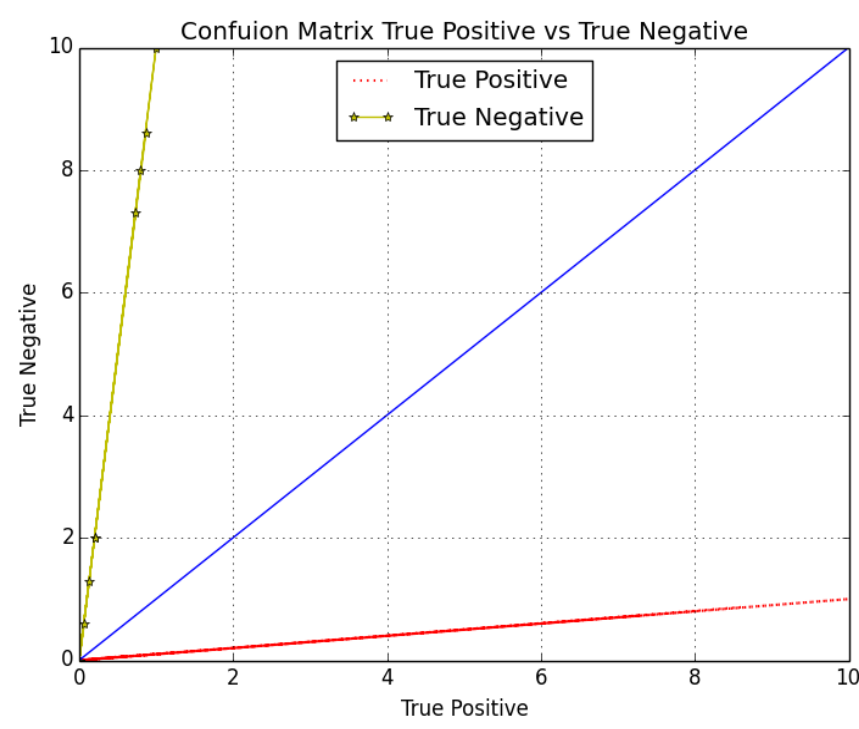

Figure 2. Confusion matrix, true positive vs true negative

\subsection{Comparative study}

In this section, related works on disease classification are compared. Table 2 shows the related works with our work and the accuracy of classification. The result of our comparative study in Table 2 shows that, the overall accuracy for different disease classification algorithms were between 68.30 and 95 percent. The accuracy of our proposed image-based hybrid approach using CNN and SVM is $85 \%$. The accuracy of the proposed system is illustrated in Figure 3. As shown in Figure 3, the accuracy of the SVM on classifying gangrene disease is between 82 and 88 percentage. This shows that, the CNN and SVM hybrid approach has better acceptable accuracy compared with other classification approaches.

Table 2. Comparative study on related proposed work

\begin{tabular}{|c|c|c|}
\hline Related and proposed work & Methodology & Accuracy \\
\hline $\begin{array}{l}\text { Classification of various skin lesion using SVM and } \\
\text { KNN classifier [8] }\end{array}$ & SVM, KNN both for classification & $\begin{array}{l}\text { SVM } 75.95 \% \\
\text { KNN } 68.30 \%\end{array}$ \\
\hline $\begin{array}{l}\text { Automated classification of liver disorders using } \\
\text { ultrasound images [9] }\end{array}$ & $\begin{array}{l}\text { Wavelet Packet Transform for segmentation, SVM } \\
\text { for Classification }\end{array}$ & $95 \%$ \\
\hline $\begin{array}{l}\text { Lung cancer detection using image processing toolbox } \\
{[10]}\end{array}$ & $\begin{array}{l}\text { For feature Extraction Gabor filter stage, Marker- } \\
\text { Controlled Watershed for Segmentation }\end{array}$ & $84.55 \%$ \\
\hline $\begin{array}{l}\text { Detection and classification of cancerous tissues in } \\
\text { digital mammograms [11] }\end{array}$ & $\begin{array}{l}\text { Gray Level Co-Occurrence Matrices (GLCMs) } \\
\text { For feature Extraction, SVM for Classification }\end{array}$ & $95 \%$ \\
\hline $\begin{array}{l}\text { Skin lesion classification from dermoscopic images } \\
\text { using deep learning techniques [20] }\end{array}$ & $\mathrm{CNN}$ for feature extraction and Classification & $78.66 \%$ \\
\hline An image-based gangrene disease classification & $\begin{array}{l}\text { CNN for Feature Extraction, SVM for } \\
\text { Classification }\end{array}$ & $85 \%$ \\
\hline
\end{tabular}


Accuracy graph of SVM gangrene disease classifier

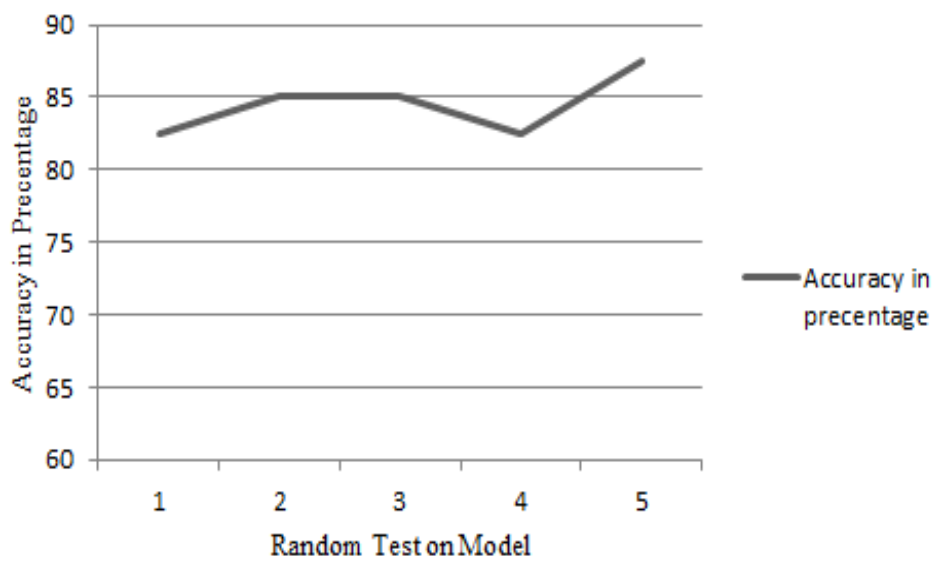

Figure 3. Accuracy of SVM gangrene disease classification

\section{CONCLUSION}

In this paper, the CNN was used for classification and feature extraction of gangrene disease images. And a multiclass support vector machine was used for the classification of images. The classification algorithm was trained with the features extracted from different images using convolutional neural network. We have applied the standard multiclass classifier using the features to classify the images into different type of Gangrene diseases. The multiclass SVM classifier was applied to achieve effective classification with multiple classes. To the greatest extent, Gangrene disease classification was automated using an image analysis technique. Using the test data, the three classes of Gangrene diseases, dry, wet, gas were classified using the SVM classifier and result analysis shown an average of 85 percent accuracy. The proposed system accuracy can be improved as we add more gangrene disease images in the training set.

\section{REFERENCES}

[1] S. S. M. Z. Ali, “GANGRENE, NIBRT-GEHC Technical Report,” pp. 1-16, 2012.

[2] B. Stapp-Caudell, "Gangrene: Recognizing and treating cellular necrosis," Association of Surgical Technologists, pp. 547-552, 2008.

[3] D. K. Srivastava and L. Bhambhu, "Data Classification using Support Vector Machine," Journal of Theoretical and Applied Information Technology, vol. 12, no. 1, pp. 1-7, 2009.

[4] T. Hastie, et al., "The Elements of Statistical Learning: Data Mining, Inference, and Prediction," Springer Series in Statistics, Springer, 2009.

[5] S. Widodo, R. N. Rohmah, B. Handaga, and L. D. D. Arini, "Lung diseases detection caused by smoking using support vector machine," TELKOMNIKA Telecommunication, Computing, Electronics and Control, vol. 17, no. 3, pp. 1256-1266, Jun. 2019.

[6] R. Priya and P. Aruna, "Review of automated diagnosis of diabetic retinopathy using the support vector machine," International Journal of Applied Engineering Research, vol. 1, no. 4, pp. 844-863, 2011.

[7] K. Crammer and Y. Singer, "On the Algorithmic Implementation of Multiclass Kernel-based Vector Machines," Journal of Machine Learning Research, vol. 2, pp. 265-292, 2001.

[8] F. Nizar and G. S. S. Kumar, "Classification of Various Skin Lesions using SVM and KNN Classifiers," International Journal of Innovative Research in Computer and Communication Engineering, vol. 4, no. 8, pp. 14710-14715, 2016.

[9] F. A. A. Minhas, et al., "Automated Classification of Liver Disorders using Ultrasound Images," Journal of Medical Systems, vol. 36, no. 5, pp. 3163-3172, 2012.

[10] A. Gaikwad, et al., "Lung cancer detection using digital Image processing On CT scan Images," International Research Journal of Engineering and Technology (IRJET), vol. 3, no. 04, pp. 2379-2381, 2016.

[11] R. Yogamangalam and B. Karthikeyan, "Segmentation Techniques Comparison in Image Processing," International Journal of Engineering and Technology (IJET), vol. 5, no. 1, pp. 307-313, 2013.

[12] D. Kaur and Y. Kaur, "Various Image Segmentation Techniques: A Review," International Journal of Computer Science and Mobile Computing (IJCSMC), vol. 3, no. 5, pp. 809-814, 2014.

[13] P. Patel and A. Thakkar, "The upsurge of deep learning for computer vision applications," International Journal of Electrical and Computer Engineering (IJECE), vol. 10, no. 1, pp. 538-548, Feb. 2020.

[14] T. A. Assegie and P. S. Nair, "Handwritten digits recognition with decision tree classification: a machine learning approach," International Journal of Electrical and Computer Engineering (IJECE), vol. 9, no. 5, pp. 4446-4451, Oct. 2019. 
[15] K. Renugambal and B. Senthilraja, "Application of Image Processing Techniques in Plant Disease Recognition," International Journal of Engineering Research \& Technology (IJERT), vol. 4, no. 3, pp. 919-923, 2015.

[16] S. B. Jadhav, et al., "Convolutional neural networks for leaf image-based plant disease classification," International Journal of Artificial Intelligence (IJ-AI), vol. 8, no. 4, pp. 328-341, Dec. 2019.

[17] H. Prasetyo, and B. A. P. Akardihas, "Batik image retrieval using convolutional neural network," TELKOMNIKA Telecommunication, Computing, Electronics and Control, vol. 17, no. 6, pp. 3010-3018, Dec. 2019.

[18] A. A. Moustafa, et al., "Optimization of deep learning features for age-invariant face recognition," International Journal of Electrical and Computer Engineering (IJECE), vol. 10, no. 2, pp. 1833-1841, Apr 2020.

[19] A. A. Abdullah, et al., "Cervical cancer detection method using an improved cellular neural network (CNN) algorithm," Indonesian Journal of Electrical Engineering and Computer Science, vol. 14, no. 1, pp. 210-218, Apr. 2019.

[20] N. Kamaruddin, A. Wahab, and Y. Rozaidi, "Neuro-physiological porn addiction detection using machine learning approach," Indonesian Journal of Electrical Engineering and Computer Science, vol. 16, no. 2, pp. 744-751, Nov. 2019.

[21] Y. Tang, "Deep Learning using Linear Support Vector Machines," International Conference on Machine Learning, Challenges in Representation Learning Workshop, Atlanta Georgia, USA, 2013.

[22] J. Nagi, "The Application of Image Processing and Machine Learning Techniques for Detection and Classification of Cancerous Tissues in Digital Mammograms," Faculty of Computer Science and Information Technology University of Malaya Kuala Lumpur, pp. 1-332, 2011.

[23] G. Dougherty, "Digital Image Processing for Medical Applications," New York, Cambridge University Press, 2009.

[24] T. Bathini, and B. Gadgay, "Hybrid enhanced ICA \& KSVM based brain tumor image segmentation" Indonesian Journal of Electrical Engineering and Computer Science, vol. 14, no. 1, pp. 478-489, Apr. 2019.

[25] A. R. Lopez, "Skin lesion detection from dermoscopic images using convolutional neural network," A Degree Thesis Submitted to the Faculty of the Escola T'ecnica d'Enginyeria de Telecomunicaci'o de Barcelona Universitat Polit`ecnica de Catalunya, 2017. 